\title{
Exploring Teaching Methods of Reliability Engineering
}

\author{
Xinli Sun ${ }^{1, a}$, Xinghui Cai ${ }^{1, b}$,Jiangren $\mathrm{Lu}^{1, \mathrm{c}}$ and Guoliang Wang ${ }^{1, \mathrm{~d}}$ \\ ${ }^{1}$ Xi'an Research Inst. of Hi-Tech, Shanxi 710025, China; \\ as_xinl@163.com, ${ }^{\mathrm{b}}$ hui_xing1975@sina.com, ${ }^{\mathrm{c}}$ ljiangren@163.com, ${ }^{\mathrm{d}}$ wgl_728@aliyun.com.
}

Keywords: Reliability Engineering; Data fusion; small sample; course construction.

Abstract. In this paper, the current teaching situation of "Reliability engineering" course for graduates of Xi'an Research Inst. of Hi-Tech are introduced. Some frequently-used data processing methods and their specific applications of graduate teaching process are presented. The relevant suggestions for concrete practice in future "Reliability engineering" course teaching are proposed.

\section{Introduction}

"Reliability engineering” is a introductory course in reliability investigation field for graduate education in Xi'an Research Inst. of Hi-Tech, Hongqing Town, Xi'an ${ }^{[1]}$. A large proportion of teaching contents are related with elementary and theoretical knowledge. During the long time teaching in these years, I realize that the course has such features as conceptions being clear, contents being arranged in proper sequence, and methods being systematic. Meanwhile, I have also found there are some problems need to be discussed. One of these problems is how to relate the graduates' future career with present course. For most graduates in my graduate school, they are going to work on the high-grade, precision and advanced equipment after leaving school. The research on these kinds of equipment has its particularity. In fact, the research on the reliability of special object will be the follow-up contents of the course of "Reliability Engineering"[2]. These follow-up contents should be learned after the graduates have grasped the general theory and method of reliability engineering. However, few students have the opportunity to further learn the contents on the application of some specific equipment. How to satisfy the graduates' requirements of the knowledge mentioned above in the teaching of the course of "Reliability Engineering"? With teaching experience, we properly add 4-6 class hour content expanding related with high-grade, precision and advanced equipment in the last few class hours. The result shows that the practice for recent several years teaching is effective. Our teaching group concludes the concrete measures and practices of the expanding with our experience in this paper.

The high-grade, precision and advanced equipment has its particularity in both data analysis and the method of evaluation.

\section{Data processing method}

\subsection{Problem}

For some product composed with a lot of different units, such large scale system product as missile and man made satellite, due to the limitation of manpower; capital and time, the system reliability experimentations for them are difficult to be conducted. Therefore, these kinds problem belong to small sample analysis problem. Meanwhile, a large quantity of unit testing information will generate in conducting the large scale complex system reliability analysis. In designing and triturating of the large scale complex system product, it is necessary to strictly conduct quality control and reliability management to ensure the reliability requirements. In a series of activities on reliability, a lot of experiment information of each unit under different conditions will be obtained. The evaluations on the reliability of the complex system precisely depend on this experiment information. From the point view of information theory, only by taking full advantage of this information can we know deeply the reliability of complex system. However, how to rationally use this experimentation information of these units under different conditions is a problem for further research ${ }^{[3]}$. Specific to the high-grade, precision and advanced equipment, this feature is very apparent. The data problems of the high-grade, 
precision and advanced equipment can be summarized into two aspects: the data source is miscellaneous and the data quantity is small.

Miscellaneous data source is in fact the problem of multi-information source. From development stage, product approval stage, to the entire service life of the high-grade, precision and advanced equipment, the quality information will be recorded. Therefore, in engineering practice, the multi-source information problem arises from the high-grade, precision and advanced equipment quality source. The parent populations, characteristic of these sources of quality information are different. In statistics, they are from different ensemble. The constraints of independence and identical distribution in classic statistics are not necessarily satisfied with. According to the classic reliability theory, the data from multi-source information should not be directly used to do reliability evaluation and research.

The quantity of some high-grade, precision and advanced equipment in service is very few. The historical data of them may not meet the bottommost requirements for reliability assessment. In reliability research, the more primary data, the more reliable conclusion will be obtained. However, in some cases, the data is too little to obtain the assessment conclusion or to result in the unreliable conclusions. This kind of problem is small sample problem. Therefore, carrying out the reliability and fusion technology research on small sample and multi-source data so as to comprehensive utilize all the quality data from high-grade, precision and advanced equipment is very necessary for us to improve the confidence level of research results.

\subsection{How to solve the problem}

\section{1) Data fusion}

Data fusion is a new technique of dealing with multi-source information in recent years. Its elementary aim is to fully apply the data from different sources to do the reliability assessment by information complementing and optimizing. Many algorithms can be applied to do the data fusion, such as Bayesian inference, Dempster-Shafer evidence theory, fuzzy set theory and neural network data fusion. However, these methods have their advantages and disadvantages. Therefore, these methods can be used in some conditions to solve specific problems and be focus on achieving the optimum result of one aspect of the dealing with the information.

There are many data fusion methods from domestic and international scholars, such as synthetic average method, Bayses estimation method, Dempster-Shafer method, Bayes method, fuzzy logic method, Product reliability test data fusion method based on parameter estimation, neural network data fusion method ${ }^{[4]}$. All of them have their own features. To take Bayes method for example, this method is mainly used to do the decision-level fusion. By synthesizing prior information and sample information into a posterior distribution, a deduction to the testing object is obtained. The merit of Bayes method is that the method may make full use of the existing information. The defect of the method is that the method is relatively sensitive to prior probability and is difficult to find out a appropriate prior distribution. In some cases, there is some prior information for the equipment. Therefore, statistic inferences based on Bayes theory are often found in some papers on the high-grade, precision and advanced equipment reliability research field. In teaching, on the base of brief introduction on some mainstream method, we mainly discuss the data fusion principle, method, procedure, applied range and features with Bayes method. In different research field, different data fusion method can be used according to the feature of research object ${ }^{[5]}$.

\section{2) Different parent distribution fusion}

Different parent distribution fusion is a kind method of data fusion, especially for the data from the high-grade, precision and advanced equipment. A lot of scholars have studied the problem of different parent and multi-source distribution under different conditions and obtained some useful conclusions. Modeling on the different distribution parameters is the main consideration for the methods of reliability testing data fusion of small sample. By now, there are some different models, such as linear model, nonlinear model, ordering model, separation of exchange variables model, can be applied to the problem. These models have their features and applicable scope. For example, linear model and nonlinear model can be applied to model the different parent distribution parameters. The process is 
simple and convenient. The information can be used fully[6]. If the modeling is precise, the data fusion effect will be favorable. But if the model is not precise enough, the fusion effect will be poor. How to improve its robustness is a problem for further study. Ordering model utilizes ordering relations among different parent distribution parameters and combines reliability testing data from different stages dealt with Bayes method to integrate and synthesize. The merits of the method are that it has a good robustness and wide application. Therefore, it can be applied to do the data fusion for such performance indexes as reliability and precision. The defect of the method is that the conclusions obtained by the integration and synthesis of the information will be partial conservative. The separation of exchange variables model applies statistics method to do the data fusion by separating the exchangeable variables from different conditions of a specific issue.

\section{3) Mechanism analysis}

The failure distribution of the material of a component can be achieved by failure mechanism analysis. Therefore, mechanism analysis may actually lay a foundation for reliability analysis.

\section{Performance degradation without failure/small sample problem}

\subsection{Problem}

Small sample problem is not only in the high-grade, precision and advanced equipment but also in the high price sophisticated product. The reasons are as follows: a. limitations to manpower (for example, earthquake prediction historic data is quite few). b. limitations to the large costs of sampling (for example, one nuclear bomb experimentation will cost to much manpower, material resources and financial resources). c. the period of sampling is too long (for example, the sample of a patient with some unusual disease may be found for several years ). The more sophisticated product, the more large cost of sampling, and the longer period of sampling, the sample is more likely to be small sample.

\subsection{How to solve the problem}

\section{1) Degradation model and forecast}

The reliability assessment method based on performance degradation analysis has been attached increasing importance with more and more high reliability product leaving factories. Compared with the traditional reliability analysis method, performance degradation analysis utilizes the data more comprehensive and may improve analysis precision to some extent. More importantly, this method may be applied in reliability without the failure of product and can remarkably shorten the testing time and reduce the sample quantity.

By now, there are two kinds reliability assessment methods based on performance degradation. One kind of the methods, such as exponential degradation orbit model, BS model and power law degradation orbit, is assumed that the performance degradation of product is subject to some given degradation orbit model. These methods can do reliability assessment with predicated product life which is predicated on the basis of estimation model parameters according to testing data. The disadvantage of the method is that the performance degradation orbit of a lot of product is usually difficult to be predicated precisely. If the performance degradation of the product is mandatorily assumed to be subject to some orbit, the error of assessment results may be relative large. If the predication of product life is precisely predicted, the assessment results may be very accurate. Another kind of the reliability assessment methods is based on performance degradation quantity distribution. This kind method assumes degradation quantity is a random quantity being subject to some distribution family. The parameter vector of the distribution family is a function of time. The solution of distribution parameters by modeling based on degradation quantity distribution may evaluate the reliability of product at specific moment. However this method may lead to accumulated computation error.

\section{2) Introduction on the method dealing with small sample}

Various methods on small sample problems have been developed, such as small sample performance degradation reliability analysis method based on gray predication, reliability assessment method based on Bayes performance degradation model. Among them, gray system theory based on 
“small sample" and "poor information" has been widely used in many fields and successfully solved many predication problems with incomplete information. Gray predication will build a GM model which may apply the past information to the future predication by the past and present known or non deterministic information, and so as to determine the future developing and changing trend of the system. Therefore, the results will be the basis of programmed decision-making. By using the gray predication, the problem of complete information and small quantity of sample will be solved in small sample performance degradation reliability analysis. This method may ensure the accuracy of pseudo life predication and obtain the reliable reliability assessment results under the condition that the degradation orbit is unknown.

\section{The problem of assessment method}

In the research on reliability assessment method, there are two common problems: one is the reliability series mode adopted by many scholars being open to discussion and another one is how to verify and fuse the results from various assessment methods.

\subsection{Discussion on reliability series mode assessment method}

\section{1) Problem}

In present assessment of reliability, the reliability series mode is often used. That is, if one or several of these numerous periodic inspection indexes of high-grade, precision and advanced equipment are a little out-of-tolerance, this equipment will be judged unqualified. This method certainly is the safest. However, the results often give us the wrong suggestions. Because even some indexes are out-of-tolerance, the equipment being evaluated may operate normally. There are two reasons for this phenomenon. (1) The parameter control margin is very large and conservative when designing the equipment. (2) Some out-of-tolerance indexes may be counterbalanced rather than superimposed during the actual operation. Some high-grade, precision and advanced equipment are still on service after several times life extensions, partly showing the present reliability series mode assessment method is not very good at reliability assessment of high-grade, precision and advanced equipment.

\section{2) How to solve the problem}

In summary, classic methods for reliability assessment are based on mathematical statistical theory and reliability theory. They process measured data and then the assessment results are acquired. Their core is using the reliability theory and based on data to acquire the assessment conclusion. This kind of methods does not take into account the operational mechanism of the product and the effect from the synthetic variations of performance parameters of each component of the product on the final indexes of the product. Actually, in many cases, the degradation of the component indexes has a cross effect on the final performance indexes of the product. This kind of cross effect can be understood only by analyzing the operation mechanism of the product. Based on this idea, we introduce our relative scientific achievements to teaching with a particular emphasis on the "reliability simulation method based on the mechanism of detonation". This method uses the numerical simulation on physical operation process to do the reliability assessment based on the analysis on the explosion process of some equipment.

\subsection{Fusion problem of multi-assessment results}

\section{1) Problem}

Usually in aircraft and naval vessel control fields, some target parameters, such as altitude, velocity, distance and position are required to be evaluated by observed data from multiple sensors. To this kind of problem, many scientific researchers may propose different mathematic models based on different ideas to solve it. Each of these models will obtain the estimation of the target parameters by using some observed data ${ }^{[6]}$. Some models may even obtain multiple estimations. Due to the different ideology of these models, the accuracy and reliability of the assessment results for a same group test sample are different. Some results of an estimation model for some samples may lose efficacy or deviate a lot. For some explicit failures, we can identify them by failure threshold, and then reject them. However, to those large implicit failures, it is difficult to overcome with single estimation model due to the lack of extra information to do the identification. 
When only two estimation models can be used, the classic confidence distance consistency detection algorithm and Bayes estimation method can not be adopted due to the too few estimation models and the unknown prior distribution of the parameters to be estimated. Therefore, it remains a problem of how to design a data fusion algorithm so as to effectively analyze the complementarities of the assessment results of the two models and to identify and reject one of the models whose estimations deviate too far and finally offer an estimation of the target parameters with higher precision and reliability.

\section{2) How to solve the problem}

Multi-model estimation fusion algorithm based on BP (Back Propagation) neural network may be a solution to the problem. BP neural network is a kind of parallel information processing model built by simulating the information processing mechanism of human brain. It has a kind of distributed storage and associative memory function. Therefore, it has a strong self-adaptivity, self-origination and arbitrary nonlinear mapping capacity so as to perform effective analysis and integration to output results from the two estimation models with high precision and reliability. Hu Wei and his group working for China Academy of Engineering Physics have carried out a research on "multi-model estimation fusion algorithm based on BP neural network" and proposed a solution to multi-result fusion.

\section{Conclusion}

In general, this kind of theoretical courses related closed with specialty is improperly to be given by common teaching method often used in basic theoretical course. It should be carried out more flexible and diverse in different forms. It should be given by combining with the interest of students on the basis of introducing relative research situation. The teaching results will be satisfactory if problem based teaching and seminar teaching are adopted.

\section{References}

[1]. Sun Xinli, Lu Changjie. Course of Engineering Reliability [M]. Beijing, National Defence Industry Press.2005

[2]. Tang Xuemei, Zhang Jinkui, Shao Fengchang, Li Rong. Analysis and Evaluation of Small Sample Test of Weapon Equipment [M]. Beijing, National Defence Industry Press.2001

[3]. Zhang Yongqiang, Liu Qi, Zhou Jinglun . Reliability Evaluation Based on Bayes Performance Degradation Model[J]. Electronic product Reliability and Environmental Testing. 2006: Vol24,NO8,

[4]. Donglin Yao. System Reliability Method and Research of Small Sample[R]. Shenyang Aerospace University. Shenyang. 2011.

[5]. Cao Xin. System Reliability Method and Research of Small Sample Based on the Bayes Performance Degradation Model[R]. The Second Artillery Engineering University. Xi'an. 2009

[6]. Liang Qingwei, Song Baowei, Shao Cheng, etc. Small-scale reliability Assessment method[J]. Machinery Design and Manufacture. 2004. 